\title{
Prolonged shedding of rhinovirus and re-infection in adults with respiratory tract illness
}

\author{
Kalina T. Zlateva', Jutte J.C. de Vries' ${ }^{1}$, Frank E.J. Coenjaerts ${ }^{2}$, Anton M. van Loon², \\ Theo Verheij ${ }^{3}$, Paul Little ${ }^{4}$, Christopher C. Butler ${ }^{5}$, Herman Goossens ${ }^{6}$, \\ Margareta leven ${ }^{6}$ and Eric C.J. Claas ${ }^{1}$ on behalf of the GRACE Study Group
}

\begin{abstract}
Affiliations: ${ }^{1}$ Dept of Medical Microbiology, Leiden University Medical Center, Leiden, The Netherlands. ${ }^{2}$ Dept of Medical Microbiology, University Medical Center Utrecht, Utrecht, The Netherlands. ${ }^{3}$ Dept of Data management, Julius Center for Health Sciences and Primary Care, University Medical Center Utrecht, Utrecht, The Netherlands. ${ }^{4}$ University of Southampton Medical School, Primary Care Medical Group, Southampton, UK. ${ }^{5}$ Dept of Primary Care Sciences, Oxford University, Oxford, UK. ${ }^{6}$ Dept of Medical Microbiology, Vaccine and Infectious Disease Institute, University Hospital Antwerp, Antwerp, Belgium.
\end{abstract}

Correspondence: Kalina T. Zlateva, Dept of Medical Microbiology, Leiden University Medical Center, Albinusdreef 2, PO Box 9600, E4-P, 2300 RC Leiden, The Netherlands. E-mail: kalina.zlatevaldgmail.com

ABSTRACT Rhinovirus infections occur frequently throughout life and have been reported in about onethird of asymptomatic cases. The clinical significance of sequential rhinovirus infections remains unclear.

To determine the incidence and clinical relevance of sequential rhinovirus detections, nasopharyngeal samples from 2485 adults with acute cough/lower respiratory illness were analysed. Patients were enrolled prospectively by general practitioners from 12 European Union countries during three consecutive years (2007-2010). Nasopharyngeal samples were collected at the initial general practitioner consultation and 28 days thereafter and symptom scores were recorded by patients over that period.

Rhinovirus RNA was detected in 444 (18\%) out of 2485 visit one samples and in $110(4.4 \%)$ out of 2485 visit two respiratory samples. $21(5 \%)$ of the 444 patients had both samples positive for rhinovirus. Genotyping of both virus detections was successful for 17 (81\%) out of 21 of these patients. Prolonged rhinovirus shedding occurred in six (35\%) out of 21 and re-infection with a different rhinovirus in 11 $(65 \%)$ out of 21 . Rhinovirus re-infections were significantly associated with chronic obstructive pulmonary disease $(p=0.04)$ and asthma $(p=0.02)$ and appeared to be more severe than prolonged infections.

Our findings indicate that in immunocompetent adults rhinovirus re-infections are more common than prolonged infections, and chronic airway comorbidities might predispose to more frequent rhinovirus re-infections.

@ERSpublications

Chronic airway comorbidities might predispose to more frequent rhinovirus re-infections in immunocompetent adults http://ow.ly/vdB3g

This article has supplementary material available from erj.ersjournals.com

Received: Oct 022013 | Accepted after revision: Jan 312014 | First published online: May 222014

Support statement: This work was supported by the $6^{\text {th }}$ Framework Programme of the European Commision (Reference: LSHM-CT-2005-518226) and the Dept of Medical Microbiology, Leiden University Medical Center, Leiden, The Netherlands.

Conflict of interest: Disclosures can be found alongside the online version of this article at erj.ersjournals.com

Copyright (OERS 2014 


\section{Introduction}

Rhinovirus (RV) is a major cause of acute respiratory disease in both children and adults. The clinical spectrum of rhinovirus infection can range from asymptomatic to more severe lower respiratory tract illness such as obliterative bronchiolitis and pneumonia [1]. Rhinovirus is recognised as a major trigger of asthma and chronic obstructive pulmonary disease (COPD) exacerbations [2]. It is also a commonly detected copathogen identified in $24 \%$ and $30 \%$ of mixed viral and bacterial infections, respectively [3, 4]. Rhinovirus infection might contribute to serious complications such as obliterative bronchiolitis and acute graft rejection in lung and stem-cell transplant recipients [5-7].

Rhinovirus is a member of the genus Enterovirus, family Picornaviridae. Currently, more than 160 sero-/ genotypes have been described and classified within three main species: RV-A, RV-B and RV-C [8]. In immunocompetent individuals, rhinovirus infections are usually associated with a mild self-limiting upper respiratory tract illness that resolves spontaneously within 1-2 weeks [9]. Virus shedding lasts on average for 10-14 days in immunocompetent subjects; however, this shedding is not always associated with respiratory symptoms $[10,11]$. Using sensitive molecular techniques, rhinovirus RNA has been detected in 10-35\% of apparently healthy subjects and therefore the clinical relevance of RT-PCR-positive results remains controversial [10, 12-14]. Prolonged rhinovirus detection for 5-6 weeks after a symptomatic infection has been reported in young children, suggesting that the virus can persist for a significant period of time. However, as no genotyping was performed, a new infection with another rhinovirus could not be excluded [15-17]. Chronic rhinovirus carriage for as long as $4-12$ months has been documented in patients with immunodeficiency after cord blood or organ transplantation $[18,19]$.

Multiple rhinovirus types co-circulate in the community and re-infections occur throughout life, suggesting that cross-protective immunity between serotypes is incomplete [11,20]. Adults experience on average two to three infections and young children up to 12 infections per year [21]. Only a few studies have evaluated the clinical severity and nature of rhinovirus re-infections and most were conducted in young children [11, 22]. It is unclear how prior rhinovirus infections modulate the clinical outcome of successive rhinovirus infections and whether certain rhinovirus types can cause more severe and prolonged illness.

The objectives of this study were: 1) to investigate the incidence of prolonged rhinovirus shedding and reinfection among adult GP patients with acute cough/lower respiratory illness; and 2) to establish the clinical severity related to prolonged rhinovirus shedding and re-infection.

\section{Methods}

Study design, subjects and specimens

Study subjects were enrolled as part of the GRACE (Genomics to combat Resistance against Antibiotics in Community-acquired lower respiratory tract infection in Europe) European Network of Excellence (www. grace-lrti.org) focusing on improving the management of community-acquired lower respiratory tract infections. 16 primary care networks from 12 European countries (Belgium, England, France, Germany, Italy, the Netherlands, Poland, Slovakia, Slovenia, Spain, Sweden and Wales) participated in a prospective recruitment of patients within the winter seasons between October 2007 and June 2010. Eligible patients were aged 18 years or older and consulting their general practitioner (GP) for the first time with a respiratory illness presenting with an acute or worsened cough ( $\leqslant 28$ days duration) as the main symptom, or any clinical presentation that suggested a lower respiratory tract infection. Exclusion criteria were pregnancy, breast-feeding and any condition associated with severe impaired immune status. Patients with previous diagnoses of asthma, COPD and other comorbid disorders, such as diabetes; respiratory, cardiovascular and allergic diseases were not excluded and thus acute infective exacerbations were included as well. The study was approved by the medical ethics committees of the participating countries. Following written informed consent, a sputum (if available) and nasopharyngeal swab sample were collected from each patient during their first visit (V1) to the GP. A follow-up nasopharyngeal swab sample was obtained at the second visit (V2) to the GP approximately 28 days later.

\section{Clinical characteristics and disease severity}

Clinical data, including past medical history, comorbidities and their management/treatment, and days the patient felt unwell were recorded by the GP on a case report form at the time of the first consultation. Following the first visit at the primary care centre, patients were requested to complete a daily symptom diary for the duration of illness (to a maximum of 28 days). The presence or absence of 13 symptoms were documented: cough, sputum production, shortness of breath, wheeze, coryza, fever, chest pain, muscle aching, headache, disturbed sleep, feeling generally unwell, interference with normal activities and confusion/disorientation. If present, the severity of each symptom was rated on a 7-point scale from 0 to 6. 0: "normal/not affected"; 1: "very little problem"; 2: "slight problem"; 3: "moderately bad"; 4: "bad"; 
5: "very bad"; and 6: "as bad as it could be". Disease severity was assessed from the following clinical outcomes: 1) duration of illness; 2) maximal symptom score; and 3) duration of higher symptom score. The duration of illness was measured as the sum of days unwell before the first GP consultation and days the patient experienced any of the 13 symptoms over the 28 -day follow-up period. The maximal symptom score was estimated as the highest mean daily symptom score based on the 13 symptoms evaluated in the patient diary during the 28-day follow-up period. The duration of higher symptom score was measured as the number of days the patient had a mean daily symptom score $\geqslant 2$ corresponding to severity ranging from "slight problem" to "as bad as it could be".

\section{Rhinovirus detection and molecular typing}

Total nucleic acids were isolated from nasopharyngeal specimens with the NucliSens EasyMag system (bioMerieux, Grenoble, France) and stored at $-70^{\circ} \mathrm{C}$ until further testing. Rhinovirus-positive samples were detected with an internally controlled real-time reverse transcriptase PCR assay amplifying a 142-bp fragment of the 5'-UTR region using primers, probes and conditions previously described [23]. Threshold cycle $(\mathrm{Ct})$ values were normalised using the same fluorescence threshold and were used as an approximate measure of viral load. Molecular typing of rhinoviruses was performed by amplification and sequencing of fragments in the VP3/VP1, VP4/VP2 or the 5'-UTR genome regions. Further information regarding the molecular typing assays and rhinovirus genotype assignment is provided in the online supplementary material.

\section{Detection of respiratory viral and bacterial co-pathogens}

Nasopharyngeal swab samples were tested by real-time PCR assays for the following viruses: human metapneumovirus (hMPV), respiratory syncytial virus (RSV), influenza viruses A and B (IFA and IFB), parainfluenza virus types 1 to 4 (PIV-1 to -4), human coronavirus (HCoV stains 229E, OC43, and NL63), adenovirus (AdV), human bocavirus ( $\mathrm{HBoV})$, polyomavirus WU (WUPyV) and KI (KIPyV) [23]. Bacterial and fungal co-infections were detected in the nasopharyngeal swab specimens by using conventional culture and/or molecular methods for Chlamydophila pneumoniae, Legionella pneumophila, Mycoplasma pneumoniae, Streptococcus spp. Haemophilus spp., Gram-negative Enterobacteriaceae, Candida sp., Aspergillus sp. and Pseudomonas aeruginosa [23]. Sputum samples were used for culture of Streptococcus pneumoniae and Haemophilus spp.

\section{Statistical analysis}

Comorbid disorders were compared per patient group using the Pearson Chi-squared or Fisher's exact test for $2 \times 2$ tables. Daily symptom scores per patient were mediated to a mean daily symptom score of the 13 symptoms. The age and clinical characteristics per patient group presented as median and interquartile range (IQR) values were compared by means of the Mann-Whitney U-test. A two-sided p-value $<0.05$ was considered statistically significant. All statistical analyses were carried out in the IBM SPSS Statistics version 20.00 software for Windows (Released 2011; IBM Corp., Armonk, NY, USA).

\section{Results}

Rhinovirus detection and molecular typing

A total of 4970 nasopharyngeal swab samples obtained from 2485 adult patients with acute cough/lower respiratory tract infection during V1 and V2 visits to the primary care centre were investigated in this study (fig. 1). Rhinovirus RNA was detected in 444 (18\%) out of 2485 V1 and 110 (4.4\%) out of 2485 V2 respiratory samples. Sequential rhinovirus detections were observed in $22(5 \%)$ out of the 444 patients with a V1 rhinovirus-positive sample. Patients with sequential rhinovirus detections included 11 males and 11 females with a median age of 55 years (range 22-78 years). To discriminate between prolonged rhinovirus shedding and re-infection, virus typing was conducted for patients with sequential rhinovirus-positive samples (figs 1 and 2). 39 (89\%) of 44 sequential rhinovirus-positive samples were successfully characterised, resulting in: RV-A $(n=22), R V-B(n=11), R V-C(n=5)$ and enterovirus (EV-C) in one case. The latter detection was due to cross-reactivity of our rhinovirus real-time RT-PCR assay with certain enterovirus types and therefore this case (patient 22) was excluded from further analysis. 12 (27\%) of the 44 samples were typed with the VP3/VP1 assay, 26 (60\%) by using the VP4/VP2 assay, and 4 (9\%) with the 5'UTR assay (see table $2 S$ in the supplementary material). Rhinovirus-positive samples that failed amplification by all three genotyping assays had significantly lower viral loads corresponding to median $\mathrm{Ct}$ value of 38 versus 29 for genotyped rhinoviruses ( $\mathrm{p}=0.002$ Mann-Whitney U-test). Both V1 and V2 rhinovirus infections could be typed for 17 (81\%) out of 21 patients, and for the remaining 4 (19\%) patients, only the first V1 sample was characterised (fig. 2). Identical rhinovirus sequences (pairwise nucleotide similarities of $99.9-100 \%)$ were found in $6(35 \%)$ out of 17 patients indicating infection with the same strain: RV-A $(n=3)$ and RV-B $(n=3)$ (table 2 S, supplementary material). In one of these patients 


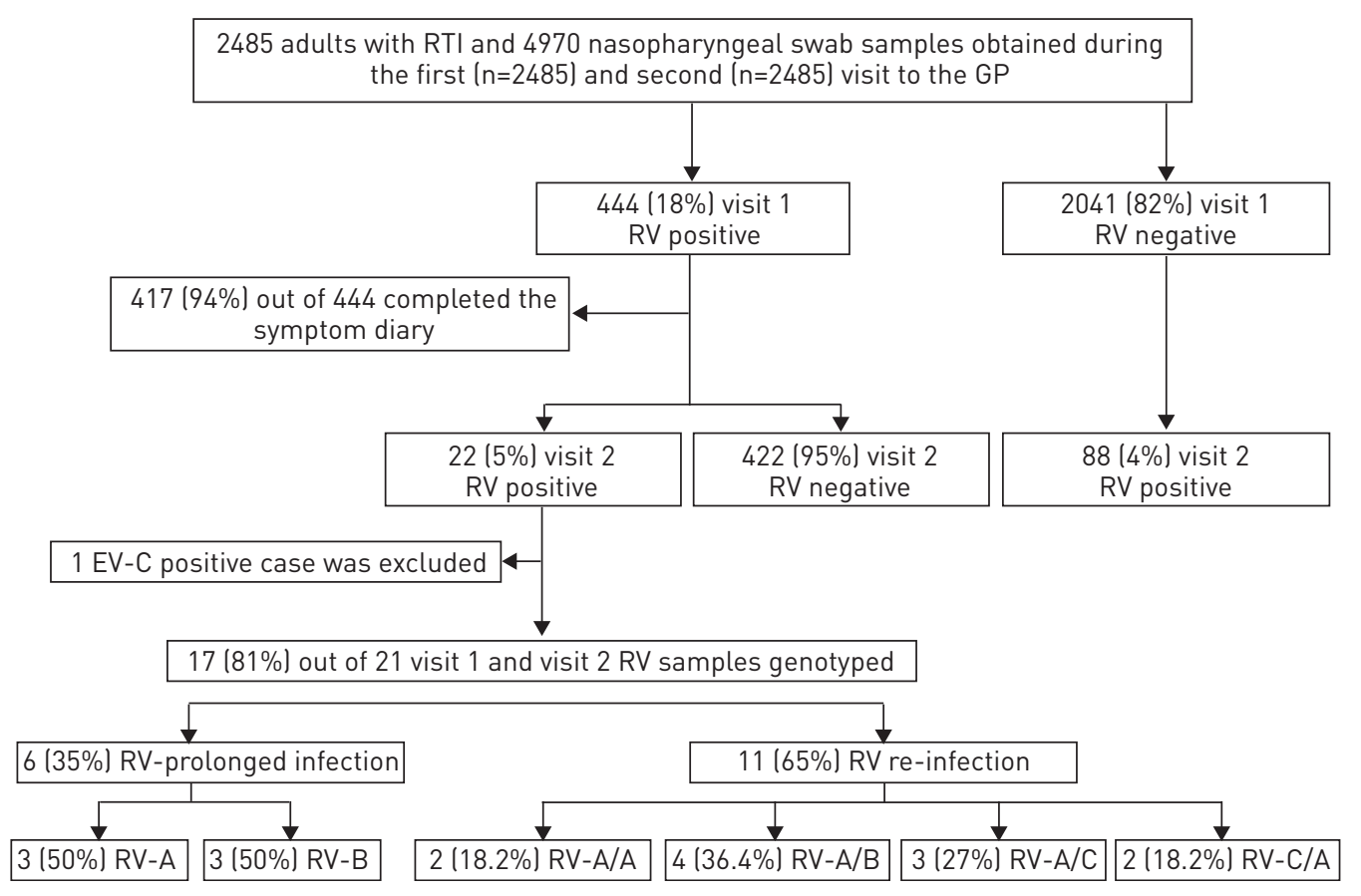

FIGURE 1 Study enrolment and results of rhinovirus (RV) testing in adults with acute cough/lower respiratory tract infection. EV-C: enterovirus C; A, B and C refer to the RV species.

(patient 6), an ambiguous nucleotide position was detected in the VP2 gene of the first V1 rhinovirus sequence (codon ATY92ATT according to RV-A43) and in another patient, a non-synonymous substitution (codon position L549V according to RV-A34) in the VP3 gene (patient 5) was observed in the second V2 virus strain.

Re-infection with a different rhinovirus strain occurred in 11 (65\%) out of 17 patients, including RV-A/RV$\mathrm{B}(\mathrm{n}=4), \mathrm{RV}-\mathrm{A} / \mathrm{RV}-\mathrm{C}(\mathrm{n}=3), \mathrm{RV}-\mathrm{C} / \mathrm{RV}-\mathrm{A}(\mathrm{n}=2)$, and two patients were re-infected with a different RV-A genotype (fig. 2).

\section{Co-pathogen detection}

One or more viral, bacterial or fungal co-pathogens were identified in 187 (34\%) of the 553 rhinoviruspositive V1 and V2 samples, consisting of 63 (11\%) viral and $119(22 \%)$ bacterial and 38 (7\%) fungal coinfections. Co-infections with another virus and/or bacteria were identified in $6(29 \%)$ of the 21 patients with sequential rhinovirus findings, of whom, nine had a co-infection at the time of the first consultation with the GP. As shown in figure 2, one to two bacteria were identified in 6 (29\%) patients with sequential rhinovirus detections including Haemophilus influenzae $(\mathrm{n}=3)$, Haemophilus parainfluenzae $(\mathrm{n}=1)$, Moraxella catarrhalis $(\mathrm{n}=1)$, Gram-negative Enterobacteriaceae $(\mathrm{n}=1)$, Candida spp. $(\mathrm{n}=1)$, Streptococcus pseudopneumoniae $(\mathrm{n}=1)$ and $S$. pneumoniae $(\mathrm{n}=1)$. Viral co-infections were observed in five patients with sequential rhinovirus findings and included RSV $(n=1), \operatorname{WUPyV}(n=1), \operatorname{HCoV}-\mathrm{NL63}(\mathrm{n}=2)$ and influenza virus $(\mathrm{n}=1)$, and one patient had a fungal co-infection with Candida spp.

\section{Comorbidities in rhinovirus infected patients}

The age distribution and rates of comorbid disorders (COPD, asthma, lung disease, cardiovascular disease, diabetes, previous hospitalisations for respiratory illness, and allergic disease) among patients with a single (V1) and sequential (V1 and V2) rhinovirus-positive samples, prolonged rhinovirus shedding and rhinovirus re-infections are shown in table $1.16(76 \%)$ of the 21 patients with sequential rhinovirus detections had at least one underlying condition, with COPD (29\%), allergic disease (29\%) and asthma (24\%) being the most common. Comorbid disorders were prevailing (10 (91\%) out of 11) in patients with rhinovirus re-infections (fig. 2b). Sequential rhinovirus detections $(p=0.004)$, specifically rhinovirus reinfections $(\mathrm{p}=0.04)$ were associated with COPD. Asthma was the second most frequent comorbid disorder that was associated with rhinovirus re-infections $(p=0.02)$. Patients with prolonged rhinovirus shedding were generally older (median age 72 years) than patients with rhinovirus single (median age 49 years; $\mathrm{p}=0.07$ ) and re-infections (median age 48 years; $\mathrm{p}=0.12$ ). 
a) RV prolonged infection

$\begin{array}{ccl}1 & 23 \text { years/M } & \begin{array}{l}\text { RV-Bpat2 } \mathrm{Ct}=34 \\ \text { H. parainfluenzae }\end{array} \\ 2 & 51 \text { years/F } & \begin{array}{l}\text { RV-B72 } \mathrm{Ct}=28 \\ \text { M. catarrhalis, GramNegEB }\end{array} \\ 3 & 67 \text { years/F } & \begin{array}{l}\text { RV-B84 } \mathrm{Ct}=25 \\ \text { Candida } \mathrm{sp} .\end{array} \\ 4 & 77 \text { years/M } & \begin{array}{l}\text { RV-A105 } \mathrm{Ct}=29 \\ \text { S. pseudopneumoniae, RSV }\end{array} \\ 5 & 78 \text { years/M } & \begin{array}{l}\text { RV-A34 } \mathrm{Ct}=29 \\ \text { H. influenzae }\end{array} \\ 6 & 78 \text { years/M } & \text { RV-A43 Ct=24 }\end{array}$

b) RV re-infection

\begin{tabular}{|c|c|c|}
\hline 7 & 22 years/M & $\mathrm{RV}-\mathrm{A} 62 \mathrm{Ct}=20$ \\
\hline 8 & 24 years/M & $\mathrm{RV}-\mathrm{A} 22 \mathrm{Ct}=28$ \\
\hline 9 & 30 years/F & $\begin{array}{l}\text { RV-C15 Ct=31 } \\
\text { HCoV-NL63 }\end{array}$ \\
\hline 10 & 33 years/F & $\begin{array}{l}\text { RV-A07 Ct=27 } \\
\text { S. pneumoniae, H. influenzae }\end{array}$ \\
\hline 11 & 34 years/F & $\mathrm{RV}-\mathrm{A} 19 \mathrm{Ct}=22$ \\
\hline 12 & 48 years/F & $\mathrm{RV}-\mathrm{C} 11 \mathrm{Ct}=18$ \\
\hline 13 & 54 years $/ M$ & $\mathrm{RV}-\mathrm{A} 30 \mathrm{Ct}=17$ \\
\hline 14 & 57 years $/ M$ & $\begin{array}{l}\text { RV-A23 Ct=35 } \\
\operatorname{lnf} A / B\end{array}$ \\
\hline 15 & 61 years/F & RV-Apat1 Ct=27 \\
\hline 16 & 69 years/F & $\begin{array}{l}\mathrm{RV}-\mathrm{A} 21 \mathrm{Ct}=28 \\
\text { H. influenzae }\end{array}$ \\
\hline 17 & 75 years/M & $R V-A 8 C t=26$ \\
\hline
\end{tabular}

c) RV sequential detections (visit 2 strains not typed)

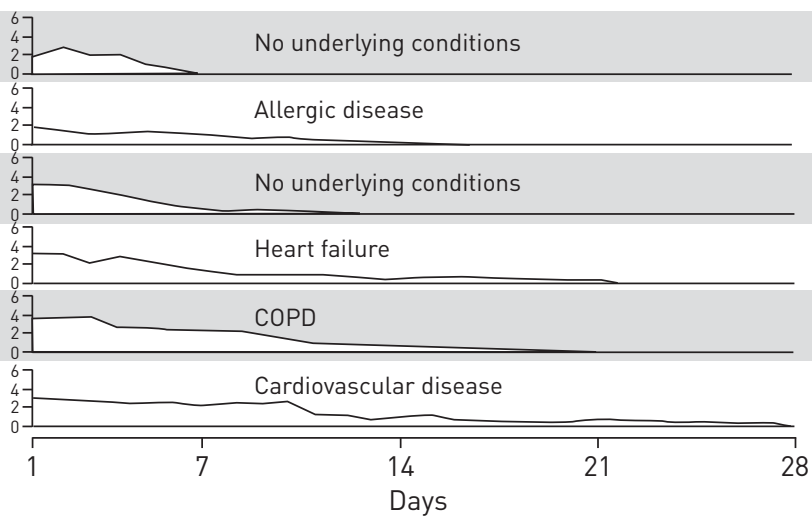

\begin{tabular}{cc} 
Allergic disease/sensitive airways \\
\hline
\end{tabular}

$\begin{array}{ll}1841 \text { years/F } & R V-A 29 \mathrm{Ct}=35 \\ 1947 \text { years/F } & R V-A 89 \mathrm{Ct}=31 \\ 2063 \text { years/M } & \text { RV-A31 Ct=30 } \\ 2172 \text { years/F } & \text { RV-B92 Ct=26 }\end{array}$

$\begin{array}{ll}\text { RV-Bpat2 } \mathrm{Ct}=28 & \text { Day } 31 \\ \text { RV-B72 } \mathrm{Ct}=34 & \text { Day } 34 \\ \text { RV-B84 Ct=28 } & \text { Day } 31 \\ \text { RV-A105 Ct=29 } & \text { Day 29 } \\ \text { RV-A34 Ct=26 } & \text { Day } 37 \\ \text { RV-A43 Ct }=40 & \text { Day } 28 \\ \text { WUPyV } & \end{array}$

RV-B72 Ct=33 Day 35

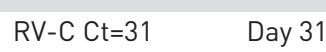

$\begin{array}{ll}\text { RV-A82 Ct=27 } & \text { Day } 39 \\ \text { RV-A2 Ct=31 } & \text { Day } 41 \\ \text { H. influenzae } & \\ \text { RV-C11 Ct=32 } & \text { Day } 29\end{array}$

RV-A46 Ct=29 Day 36

$\mathrm{RV}-\mathrm{C} 15 \mathrm{Ct}=30 \quad$ Day 45

RV-B92 Ct=35 Day 40

RV-B72 Ct=32 Day 36

RV-B70 Ct=22 Day 31

RV-A2 Ct=37 Day 45

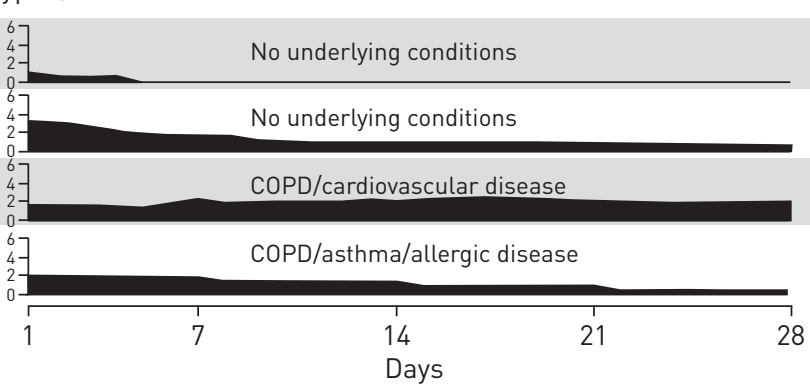

$\begin{array}{ll}\text { RV Ct }=28 & \text { Day } 36 \\ \text { HCoV-NL63 } & \text { Day } 36 \\ \text { RV Ct=36 } & \text { Day } 31 \\ \text { RV Ct=32 Ct=40 } & \text { Day } 35 \\ & \end{array}$

FIGURE 2 Diagnosis and daily symptoms scores of patients with sequential rhinovirus-positive samples during a 28-day follow-up period. a) Patients with prolonged rhinovirus shedding, b) rhinovirus re-infections and c) not typed rhinovirus sequential findings. Rhinovirus genotypes with respective cycle threshold (Ct) values and co-pathogens identified in visit 1 (day 1) and visit 2 (day 28 or later) samples are shown. Potentially novel rhinovirus genotypes are indicated as provisionally assigned types (pat). Underlying conditions and relevant medical history are specified above the corresponding daily symptom graph of each patient. M: male; F: female; H. parainfluenza: Haemophilus parainfluenzae; M. catarrhalis: Moraxella catarrhalis; GramNegEB: Gram-negative Enterobacteriaceae; S. pseudopneumoniae: Streptococcus pseudopneumoniae; RSV: respiratory syncytial virus; H. influenzae: Haemophilus influenzae; COPD: chronic obstructive pulmonary disease; S. pneumoniae: Streptococcus pneumoniae; HCV: hepatitis C virus; RTI: respiratory tract infection. 

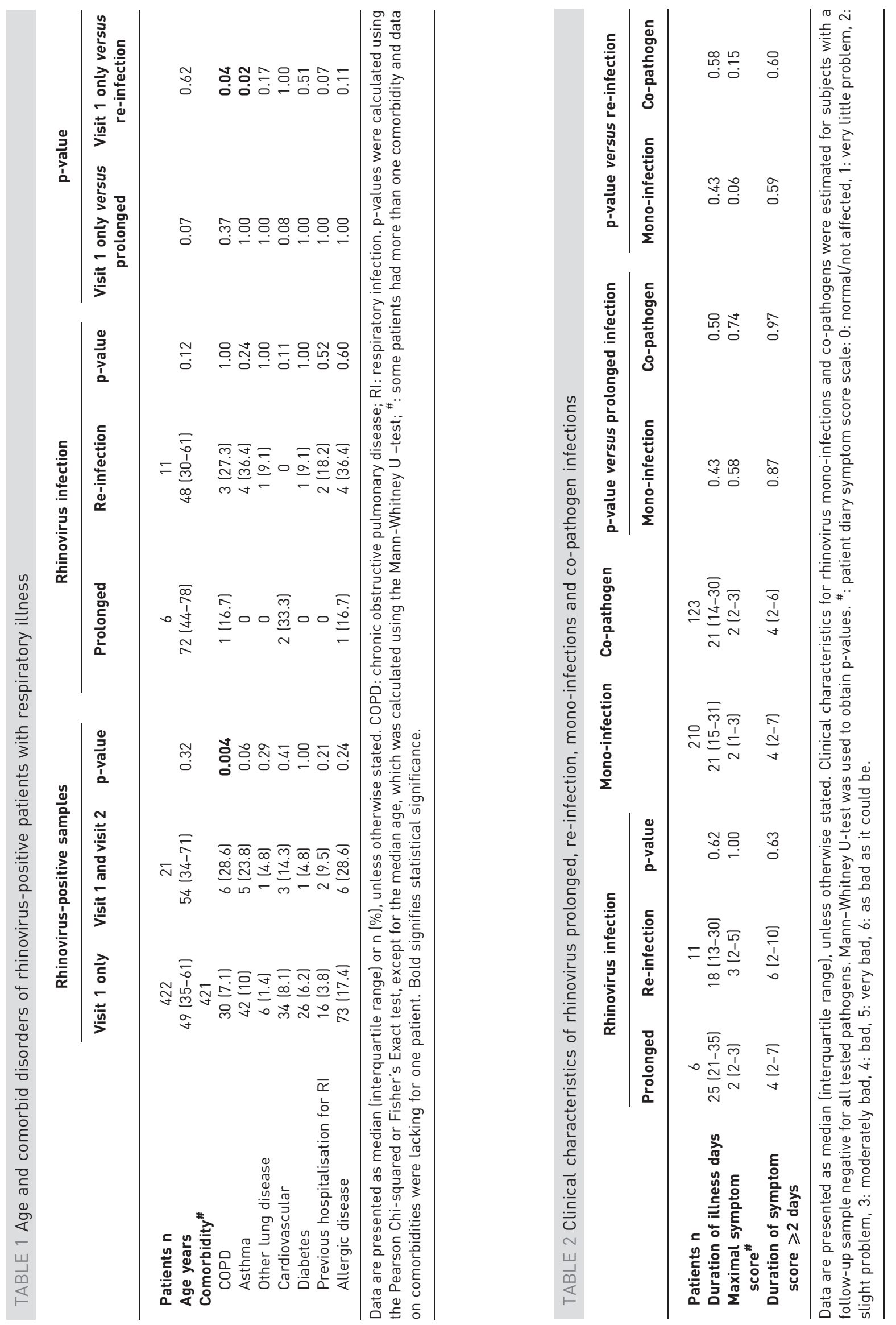
Clinical evaluation of prolonged rhinovirus shedding and re-infection

For patients with sequential rhinovirus detection, the second follow-up sample was obtained 28 days or later after the first V1 respiratory sampling; median (IQR) 35 days (31-40 days). 12 (57\%) of the 21 patients with sequential rhinovirus-positive samples had no clinical symptoms on day 28 following the first V1 rhinovirus detection, seven $(33 \%)$ patients had "very little problem" (symptom scores $\leqslant 1.2$ ), and two (9\%) patients had "slight problem" (symptom scores $\sim 2$ ). The clinical characteristics of rhinovirus prolonged and reinfections are presented in table 2. The median duration of illness was longer for patients with a prolonged infection (25 versus 18 days), but the median maximal symptom score and median duration of symptoms $\geqslant 2$, were higher for rhinovirus re-infections. The disease severity associated with prolonged rhinovirus shedding and rhinovirus re-infection was also compared to that of 210 cases with rhinovirus monoinfection and 123 cases with co-infections with other viral or bacterial pathogens (table 2). The clinical features of the latter were estimated only for rhinovirus V1 positive patients that had a V2 follow-up sample negative for all screened pathogens, thus reducing biases in rhinovirus symptom severity due to a sequential infection with another pathogen. The analysis showed no significant differences between the symptom severities of prolonged rhinovirus shedding and rhinovirus re-infections, as well as when compared with rhinovirus mono- and co-pathogen infections.

\section{Discussion}

In the present study, rhinovirus was detected in $18 \%$ of immunocompetent adults with acute cough or any other reason for suspecting a lower respiratory tract infection. A follow-up sample obtained approximately 28 days later was rhinovirus positive in $5 \%$ of adults with initial rhinovirus infection, representing $0.9 \%$ of the total patient cohort. Rhinovirus genotyping revealed that the majority $(65 \%)$ of subjects were reinfected with a different rhinovirus (fig. 1). In contrast to previous studies that have identified RV-B infections less frequently in children with respiratory tract illness [20,24], in the current study, RV-B was the second most common species after RV-A and was identified in three out of the six patients with prolonged rhinovirus shedding.

More than half of the patients with sequential rhinovirus detections had no symptoms (57\%) or very mild symptoms (33\%) at the time (day 28) or on average 7 days before the second virus detection (fig. 2). These data suggest that, in adults, follow-up rhinovirus infections are likely to be mild or asymptomatic. Our observations are consistent with previous findings of asymptomatic secondary rhinovirus infections among adults [10]. In immunocompetent individuals, the acquired immunity to previous rhinovirus exposures determines the clinical severity and duration of subsequent rhinovirus infections. High titers of serotypespecific antibodies in serum and respiratory secretions have been associated with reduced symptom severity or resistance to homologous rhinovirus infections [25]. In addition, broadly cross-reactive neutralising antibodies raised against conserved peptide sequences in the VP4, VP3 and VP1 capsid proteins have been previously reported $[26,27]$.

In the current study, most patients $(77 \%)$ with sequential rhinovirus detections had at least one comorbid disorder; with COPD, allergic disease and asthma accounting for the majority of cases. Furthermore rhinovirus re-infections were significantly associated with COPD and asthma comorbidities indicating that chronic airway disorders might predispose to more frequent rhinovirus infections. A recent study conducted on asthmatic children hospitalised for severe exacerbations has shown that repeated rhinovirus detection over 8 weeks in eight patients was due to re-infection [24]. In this study, re-infection was confirmed in 11 of the 17 patients with genotyped rhinovirus positive follow-up samples. Our findings provide further evidence that sequential rhinovirus detections are predominantly re-infection rather than persistence.

MOLYNEAUX et al. [28] demonstrated that in contrast to healthy individuals rhinovirus infection in subjects with COPD leads to a significant outgrowth of potentially pathogenic $H$. influenza. In that study, bacterial co-pathogens were identified in $29 \%$ ( 6 out of 21) of the patients with recurrent rhinovirus detections with $H$. influenzae being the most frequently observed bacterial species identified in three patients. All three patients had underlying respiratory tract conditions, including two cases with COPD and one patient had a previous hospitalisation for pleural tuberculosis.

In immunocompetent adults, the duration of rhinovirus shedding is usually 1-2 weeks and prolonged rhinovirus infection over 28 days has been reported only for patients with severe immune deficiencies $[11,18,19]$. In our study, four of the six adults who shed rhinovirus for over 28 days were older than 65 years. Although the sample size was underpowered for significance testing, these patients were in general older and had a longer duration of illness in comparison with patients with rhinovirus re-infections, monoinfections and rhinovirus co-pathogen infections (table 1 and 2). Prolonged infections were caused by various RV-A and RV-B genotypes and the viral loads at the time of the first (V1) and second (V2) rhinovirus detection were comparable and relatively high for the majority of patients, indicating persistent 
viral replication (fig. 2a). Age-related changes in the innate and adaptive immune responses such as diminished antibacterial activity of neutrophils, decline in cytokine production, and antibodies exhibiting lower affinity and avidity for antigen have been reported [29]. Immunosenescence may therefore lead to poor viral clearance and more frequent and severe respiratory infections in the elderly. Importantly, the presence of synonymous and non-synonymous changes in the VP2 and VP3 capsid genes in two RV-A strains suggests that the elderly may represent a potential reservoir for the emergence and spread of rhinovirus variants with modified antigenic properties.

In our study, rhinovirus re-infected patients did not experience apparent aggravation of disease symptoms during the 28-day follow-up period (fig. 2b). Still, the overall clinical manifestation appeared to be more severe than prolonged rhinovirus infections, presenting with higher symptom scores and a longer duration of more severe symptoms. The high rate of respiratory comorbidities in these patients could possibly explain the more severe disease outcome.

The current study has certain limitations. Since a good-quality sputum sample is difficult to obtain, particularly from older patients [30], pathogen screening was performed mainly on nasopharyngeal swab specimens. Another limitation is that sampling was not conducted on a more frequent (weekly) basis; thus, the time of the second rhinovirus infection cannot be precisely determined. Further research is needed to establish if sequential rhinovirus infections, particularly with a different rhinovirus species can lead to more severe symptoms.

To our knowledge, this represents the first extensive population-based study on the incidence and clinical relevance of repeated rhinovirus detections among healthy adults. The data presented here indicate that in immunocompetent adults sequential rhinovirus infections are primarily due to re-infection with a different rhinovirus genotype. Our findings suggest that patients with chronic airway comorbidities may have increased susceptibility to rhinovirus re-infection, while prolonged rhinovirus shedding may be more common in the elderly. The role of advanced age and immunosenescence in prolonged rhinovirus shedding and transmission warrants further investigation.

\section{Acknowledgements}

We thank all involved in the GRACE Primary Care Network for the opportunity to conduct this study, Jacqueline Schelfault, Yvette van Aarle, Lopje Höcker and Lisette Rusman (Dep. of Medical Microbiology, LUMC, The Netherlands) for excellent technical assistance and Susan Hemert-Glaubitz (Julius Center for Health Sciences and Primary Care, UMC, The Netherlands) for assistance with the GRACE database, Peter Simmonds and Chloe McIntyre (Roslin Institute, University of Edinburgh, UK) for assistance with rhinovirus type classification.

\section{References}

Jacobs SE, Lamson DM, St GK, et al. Human rhinoviruses. Clin Microbiol Rev 2013; 26: 135-162.

Gern JE. The ABCs of rhinoviruses, wheezing, and asthma. J Virol 2010; 84: 7418-7426.

Greer RM, McErlean P, Arden KE, et al. Do rhinoviruses reduce the probability of viral co-detection during acute respiratory tract infections? J Clin Virol 2009; 45: 10-15.

4 Honkinen M, Lahti E, Osterback R, et al. Viruses and bacteria in sputum samples of children with communityacquired pneumonia. Clin Microbiol Infect 2012; 18: 300-307.

5 Costa C, Bergallo M, Astegiano S, et al. Detection of human rhinoviruses in the lower respiratory tract of lung transplant recipients. Arch Virol 2011; 156: 1439-1443.

6 Kumar D, Husain S, Chen MH, et al. A prospective molecular surveillance study evaluating the clinical impact of community-acquired respiratory viruses in lung transplant recipients. Transplantation 2010; 89: 1028-1033.

7 Versluys $\mathrm{AB}$, Rossen JW, van $\mathrm{EB}$, et al. Strong association between respiratory viral infection early after hematopoietic stem cell transplantation and the development of life-threatening acute and chronic alloimmune lung syndromes. Biol Blood Marrow Transplant 2010; 16: 782-791.

8 McIntyre CL, Knowles NJ, Simmonds P. Proposals for the classification of human rhinovirus species A, B and C into genotypically assigned types. J Gen Virol 2013; 94: 1791-1806.

9 Gwaltney JM. Clinical significance and pathogenesis of viral respiratory infections. Am J Med 2002; 112: Suppl. 6A, 13S-18S.

10 Peltola V, Waris M, Osterback R, et al. Rhinovirus transmission within families with children: incidence of symptomatic and asymptomatic infections. J Infect Dis 2008; 197: 382-389.

11 Peltola V, Waris M, Kainulainen L, et al. Virus shedding after human rhinovirus infection in children, adults and patients with hypogammaglobulinaemia. Clin Microbiol Infect 2013; 19: E322-E327.

12 Johnston SL, Sanderson G, Pattemore PK, et al. Use of polymerase chain reaction for diagnosis of picornavirus infection in subjects with and without respiratory symptoms. J Clin Microbiol 1993; 31: 111-117.

13 van Benten I, Koopman L, Niesters B, et al. Predominance of rhinovirus in the nose of symptomatic and asymptomatic infants. Pediatr Allergy Immunol 2003; 14: 363-370.

14 van Gageldonk-Lafeber AB, Heijnen ML, Bartelds AI, et al. A case-control study of acute respiratory tract infection in general practice patients in The Netherlands. Clin Infect Dis 2005; 41: 490-497.

15 Jartti T, Lehtinen P, Vuorinen T, et al. Persistence of rhinovirus and enterovirus RNA after acute respiratory illness in children. J Med Virol 2004; 72: 695-699.

16 Kling S, Donninger H, Williams Z, et al. Persistence of rhinovirus RNA after asthma exacerbation in children. Clin Exp Allergy 2005; 35: 672-678. 
17 Winther B, Hayden FG, Hendley JO. Picornavirus infections in children diagnosed by RT-PCR during longitudinal surveillance with weekly sampling: Association with symptomatic illness and effect of season. J Med Virol 2006; 78: 644-650.

18 Kaiser L, Aubert JD, Pache JC, et al. Chronic rhinoviral infection in lung transplant recipients. Am J Respir Crit Care Med 2006; 174: 1392-1399.

19 Pathak AK, Adams RH, Shah NC, et al. Persistent human rhinovirus type C infection of the lower respiratory tract in a pediatric cord blood transplant recipient. Bone Marrow Transplant 2013; 48: 747-748.

20 Sansone M, Andersson M, Brittain-Long R, et al. Rhinovirus infections in western Sweden: a four-year molecular epidemiology study comparing local and globally appearing types. Eur J Clin Microbiol Infect Dis 2013; 32: 947-954.

21 Turner RB. Epidemiology, pathogenesis, and treatment of the common cold. Ann Allergy Asthma Immunol 1997; 78: 531-539.

22 Jartti T, Lee WM, Pappas T, et al. Serial viral infections in infants with recurrent respiratory illnesses. Eur Respir J 2008; 32: 314-320.

23 Loens K, van Loon AM, Coenjaerts F, et al. Performance of different mono- and multiplex nucleic acid amplification tests on a multipathogen external quality assessment panel. J Clin Microbiol 2012; 50: 977-987.

24 Engelmann I, Mordacq C, Gosset P, et al. Rhinovirus and asthma: reinfection, not persistence. Am J Respir Crit Care Med 2013; 188: 1165-1167.

25 Alper CM, Doyle WJ, Skoner DP, et al. Prechallenge antibodies moderate disease expression in adults experimentally exposed to rhinovirus strain hanks. Clin Infect Dis 1998; 27: 119-128.

26 Katpally U, Fu TM, Freed DC, et al. Antibodies to the buried N terminus of rhinovirus VP4 exhibit cross-serotypic neutralization. J Virol 2009; 83: 7040-7048.

27 McCray J, Werner G. Different rhinovirus serotypes neutralized by antipeptide antibodies. Nature 1987; 329: 736-738.

28 Molyneaux PL, Mallia P, Cox MJ, et al. Outgrowth of the bacterial airway microbiome after rhinovirus exacerbation of chronic obstructive pulmonary disease. Am J Respir Crit Care Med 2013; 188: 1224-1231.

29 Meyer KC. The role of immunity and inflammation in lung senescence and susceptibility to infection in the elderly. Semin Respir Crit Care Med 2010; 31: 561-574.

30 Loens K, Van HL, Malhotra-Kumar S, et al. Optimal sampling sites and methods for detection of pathogens possibly causing community-acquired lower respiratory tract infections. J Clin Microbiol 2009; 47: 21-31. 